Article

\title{
The DNA-Damage Response to $\gamma$-Radiation Is Affected by miR-27a in A549 Cells
}

\author{
Andrea Di Francesco ${ }^{1, \dagger}$, Cristiano De Pittà ${ }^{1, \dagger}$, Francesca Moret ${ }^{1}$, Vito Barbieri ${ }^{2}$, \\ Lucia Celotti $^{1,3, *}$ and Maddalena Mognato ${ }^{1, *}$
}

1 Department of Biology, University of Padova, via U. Bassi 58/B, Padova 35131, Italy;

E-Mails: difrancesco_andrea@hotmail.it (A.D.F.); cristiano.depitta@unipd.it (C.D.P.); francesca.moret@unipd.it (F.M.)

2 Department of Surgery, Oncology and Gastroenterology, University of Padova via Gattamelata 64, Padova 35128, Italy; E-Mail: vito.barbieri@ unipd.it

3 INFN-Laboratori Nazionali di Legnaro, Viale dell’Università 2, Legnaro 35020, Padova, Italy

$\dagger$ These authors contributed equally to this work.

* Authors to whom correspondence should be addressed; E-Mails: lucia.celotti@unipd.it (L.C.); maddalena.mognato@unipd.it (M.M.); Tel.: +39-049-8276-283 (L.C.); +39-049-8276-286 (M.M.); Fax: +39-049-8276-280 (L.C. \& M.M.).

Received: 17 June 2013; in revised form: 2 August 2013 / Accepted: 7 August 2013 /

Published: 2 September 2013

\begin{abstract}
Perturbations during the cell DNA-Damage Response (DDR) can originate from alteration in the functionality of the microRNA-mediated gene regulation, being microRNAs (miRNAs), small non-coding RNAs that act as post-transcriptional regulators of gene expression. The oncogenic miR-27a is over-expressed in several tumors and, in the present study, we investigated its interaction with ATM, the gene coding for the main kinase of DDR pathway. Experimental validation to confirm miR-27a as a direct regulator of ATM was performed by site-direct mutagenesis of the luciferase reporter vector containing the 3'UTR of ATM gene, and by miRNA oligonucleotide mimics. We then explored the functional miR-27a/ATM interaction under biological conditions, i.e., during the response of A549 cells to ionizing radiation (IR) exposure. To evaluate if miR-27a over-expression affects IR-induced DDR activation in A549 cells we determined cell survival, cell cycle progression and DNA double-strand break (DSB) repair. Our results show that up-regulation of miR-27a promotes cell proliferation of non-irradiated and
\end{abstract}


irradiated cells. Moreover, increased expression of endogenous mature miR-27a in A549 cells affects DBS rejoining kinetics early after irradiation.

Keywords: $\gamma$-radiation; DNA Damage Response; miR-27a; ATM; A549 cells

\section{Introduction}

To maintain the integrity of genome, eukaryotic cells rely on a highly regulated system pathway of response to DNA damage - the DNA-Damage Response (DDR) — which encompasses damage sensors, mediators, signal transducers and effectors. Upon recognition of DNA damage, transducer kinases ATM (Ataxia-Telangiectasia Mutated), ATR (Ataxia telangiectasia and Rad3 related), and DNA-PKcs (DNA-dependent protein kinase catalytic subunit) relay and amplify the damage signal to effector proteins that in turn activate cell cycle checkpoints, regulate transcription, translation, and metabolism, and activate the appropriate DNA repair process, as well as cell fate toward apoptosis or senescence [1,2]. Following DNA double-strand break (DSB) induction, ATM undergoes spatial relocalization and catalytic activation; after that, it is rapidly recruited to DNA damage sites. Here ATM phosphorylates specific serines or threonines on many downstream protein substrates, including "Ser-139" of histone variant H2AXpresent in nucleosomes surrounding DSB sites, thereby regulating DDR mechanism.

Following genotoxic stress, DDR pathway is post-transcriptionally regulated through selective mRNA stabilization or decay and regulation of translation [3]. In this context, non-coding RNAs, such as microRNAs (miRNAs), have emerged as important regulators of gene expression of key components of DDR pathway. miRNAs are natural single-stranded, small RNA molecules (18-22 nt) that regulate gene expression by binding to target mRNAs and suppress their translation or promote their cleavage [4]. Mature miRNAs recognize their target mRNAs by base-pairing interactions between nucleotides $2-8$ of the miRNA (the seed region) and complementary nucleotides in the 3'-untranslated region (3'UTR) of mRNAs. Recent evidences, however, suggest that in addition to 3'UTRs miRNAs can bind to other regions of target mRNAs, including the 5'UTRs, promoter, and open reading frames [5]. In humans, $~ 800$ miRNAs are predicted to exist [6], and each single miRNA can influence the expression of up 1000 genes. miRNAs regulate many physiological processes, including differentiation, apoptosis, fat metabolism, as well as pathological processes, such as tumorigenesis. Different miRNAs are indeed dysregulated in human cancers and can function as either tumor suppressors or oncogenes, by targeting different steps of the tumorigenesis process, including initiation and progression to a metastatic phenotype [7-10]. Moreover, accumulating evidences have shown that miRNAs are altered following genotoxic and cytotoxic stress, and several studies suggested that miRNA expression is regulated in DDR at the transcriptional level, in a p53-dependent manner [11] and through modulation of miRNAs' processing and maturation steps [12]. Moreover, more than half of the DNA repair and DNA damage checkpoint genes contained conserved miRNA target sites [13].

By integrating the transcriptome and microRNome, we recently identified some miRNA-related genes of DDR pathway that were altered in human peripheral blood lymphocytes irradiated with $\gamma$-rays and incubated in ground gravity $(1 \mathrm{~g})$ and in modeled microgravity (MMG) [14]. Several miRNAs 
were specifically dysregulated by IR in a dose-, time- and gravity-related manner. Among miRNAs altered by the combined action of radiation and microgravity, we identified miR-27a, which as a result, was anti-correlated to ATM. miR-27a is classified as an oncomir, being over-expressed in several malignancies, including breast cancer [15], gastric and renal carcinoma [16,17], hepatocellular carcinoma [18] and pediatric B-ALL [19]. Down-regulation of miR-27a has been reported in colorectal cancer [20], in oral squamous carcinoma [21], and in the serum and plasma of non-small cell lung cancer patients [22]. miR-27a is part of a cluster of three miRNAs expressed from an intergenic region of chromosome 19, whose members-miR-23a, miR-27a, miR-24-2 - are involved in cell cycle control and differentiation in various cell types [23]. Recent evidences report that miR-23a 24-2 27a cluster may possess a causal role in mammary tumorigenesis, since the expression levels of its members were significantly higher in breast cancer with lymphnode metastasis compared with that from patients without lymphnode metastasis or normal tissue [24]. The increased expression of miR-23a 24-2 27a cluster possesses also important function in neovascular age-related macular degeneration and tumor-related angiogenesis [25], however, the mechanisms of regulation in cancer progression is still poorly understood.

In the current study, we investigated the role of miR-27a in the DDR induced by $\gamma$-radiation in lung cancer A549 cells. We first validated the functional interaction between miR-27a and ATM by performing site-directed mutagenesis of 3'-untranslated region (UTR) of ATM gene. We then analyzed the biological effects of miR-27a over-expression on the DNA damage response to $\gamma$-rays in A549 cells by using miRNA mimics which are chemically synthesized double stranded RNAs that, when introduced into cells, efficiently mimic specific endogenous miRNAs.

\section{Results and Discussion}

\section{1. miR-27a Interacts with 3'UTR of ATM Gene}

ATM-mediated DNA damage response is an important barrier to prevent tumorigenesis, indeed ATM down-regulation has been observed in many cancers [26]. We recently demonstrated that miR-27a and ATM are differentially expressed and anti-correlated in $\gamma$-irradiated human lymphocytes incubated in microgravity and that miR-27a could interact with ATM-3'UTR [14]. However, to validate that miR-27a actually targets ATM-UTR, in the present study we created luciferase constructs containing wild type and mutated 3'UTR of ATM gene, and tested them for miR-27a-ATM interaction. The ATM gene has a long $(\sim 3.7 \mathrm{~kb})$ and highly conserved 3'UTR, and two different putative miR-27a target sites have been predicted by PITA algorithm. We generated two mutations in the ATM-3'UTR sequence in the complementary site for the seed region of miR-27a as indicated in Figure 1a. We co-transfected A549 cells with miR-27a precursor (pre-miR-27a) together with the wild-type ATM-3'UTR luciferase reporter or with reporter in which the $A T M-3$ 'UTR sequence was mutated at each individual miR-27a binding site (ATM-del1 and $A T M$-del2). In addition, we generated a construct containing the synthetic sequence including the perfect miR-27a binding site (sensor) to be used as positive control for each experiment (Figure 1b). As shown in Figure 1c, the activity of the reporter construct containing wild type ATM-3'UTR was significantly decreased by the treatment with miR-27a mimic, while the construct with two seed regions of miR-27a mutated at site 1 was refractory to miR-27a-mediated 
repression. By analyzing the effect of the two different mutated seed regions on luciferase activity we observed that the miR-27a binding mutated site 1 (ATM-del1) showed a real interaction with miR-27a. On the contrary, the miR-27a binding mutated site 2 (ATM-del2) showed a decreased luciferase activity, indicating a weak miRNA-mRNA interaction in that position of ATM-3'UTR sequence.

Figure 1. Ataxia-Telangiectasia Mutated $(A T M)$ is a target of miR-27a in A549 cells. (a) Diagram illustrating the two putative regions of ATM-3'UTR for miR-27a binding and corresponding mutant miR-27a binding sites. Two deletions were generated in the ATM-3'UTR sequence in the complementary site for the seed regions of miR-27a as indicated; (b) Reporter constructs containing a synthetic sequence including three perfect miR-27a binding sites (miR-27a sensor) were generated as described in the Experimental Section; (c) Effects of miR-27a binding sites on luciferase activity. A459 cells were co-transfected with the firefly luciferase reporter plasmid containing wild-type or mutant ATM-3'UTR, with pre-miRNA precursor (pre-miR-27a) or pre-miRNA precursor-Negative Control (pre-control). Luciferase activity was assayed $24 \mathrm{~h}$ after transfection. The data represent mean \pm S.D. from three independent experiments, normalized on Renilla Luciferase activity $(* * * p<0.001, t$-test); del $=$ deletion; 1 and 2 binding sites. Data of relative luciferase activity of $A T M \mathrm{wt}$ and miR-27a sensor are from Girardi et al. [14].

(a)

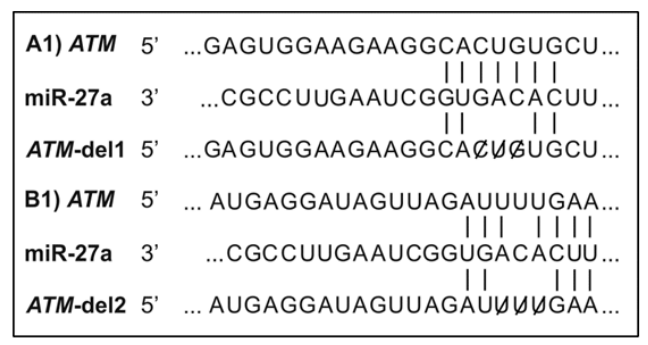

(b)

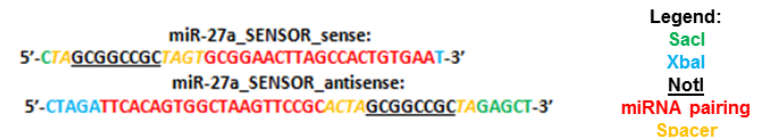

(c)

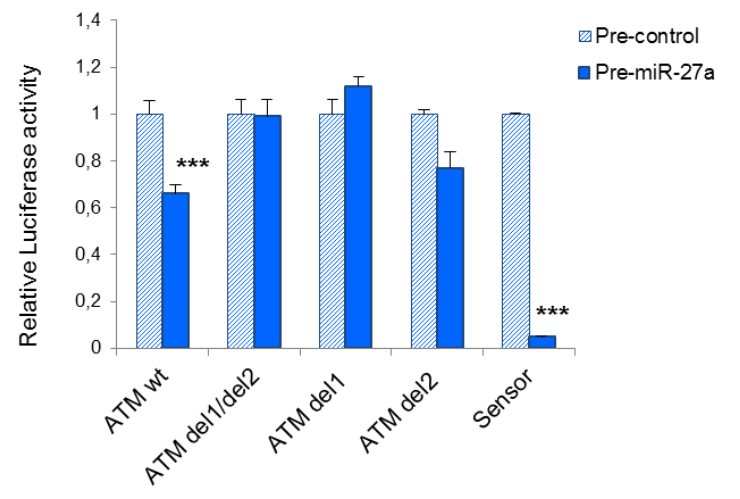

\section{2. miR-27a Affects ATM Expression Levels in A549 Cells}

The main mechanism of miRNA action is thought to be degradation of mRNA or inhibition of translation [4]; therefore, the effect of a miRNA mimic can be assayed at the mRNA level of its target gene. Before evaluating the action of miR-27a on modulation of ATM transcript, we first verified that 
the miRNA was not endogenously over-expressed in A549 cells and it was properly processed following transient transfection with plasmid and miRNA mimic. The results of quantitative RT-PCR (qRT-PCR) showed that under physiological conditions the mature miR-27a was not up-regulated in A549 cells, whereas it was highly induced after transfection (Figure 2a). At the same time, we verified if IR induced dysregulation of miR-27a by examining its expression level in $\gamma$-irradiated cells. The results show that the expression level of miR-27a is almost unaffected in 2 Gy-treated cells and only slightly increased in 5 Gy-treated cells.

Figure 2. Expression of miR-27a and $A T M$ in A549 cells. (a) Relative expression of miR-27a measured by qRT-PCR in untransfected cells (CTR), in mock-transfected cells, in miR-27a transfected cells (mimic) and in untransfected cells irradiated with $\gamma$-rays ( 2 and 5 Gy). Analyses have been performed at $24 \mathrm{~h}$ after transfection or irradiation; (b) Relative expression of ATM transcript measured by qRT-PCR in cells transfected or not with miR-27a and irradiated with 2 Gy. Analysis was performed at $5 \mathrm{~h}$ and $24 \mathrm{~h}$ after irradiation andRNU48 was used as an internal loading control in all reactions. At both time points, the expression level of ATM is significantly down-regulated in cells over-expressing miR-27a (*** $p<0.001, t$-test).

(a)

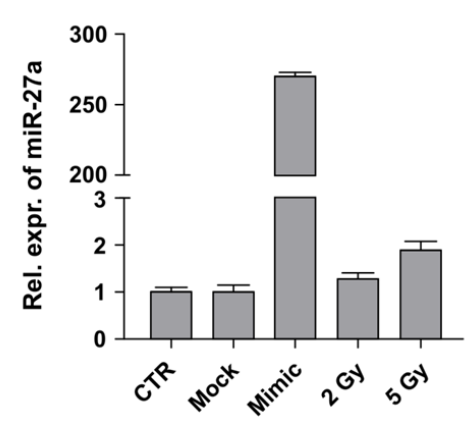

(b)

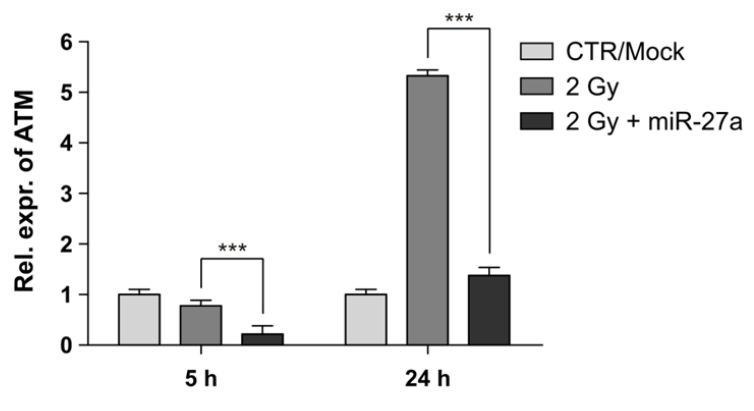

The functional interaction between miR-27a and $A T M$ transcript was validated by measuring the expression level of ATM in non-irradiated and 2 Gy-irradiated A549 cells transfected with miR-27a mimic (Figure 2b). Cells were harvested at $5 \mathrm{~h}$ and $24 \mathrm{~h}$ after irradiation and the expression level of ATM was compared with that of non-irradiated control cells. At $5 \mathrm{~h}$ after irradiation, ATM expression was slightly decreased in irradiated cells in respect to control cells, in accordance with data of Ghosh et al. [27] obtained in A549 cells irradiated with the same dose ( 2 Gy) of $\gamma$-rays. At $24 \mathrm{~h}$ after irradiation, ATM expression significantly increased ( $\sim 5$-fold) in comparison with control cells. At both time points after irradiation, notably, the level of $A T M$ was significantly decreased in cells over-expressing miR-27a. Besides the action of miR-27a on ATM-3'UTR, such mRNA reduction could be due to an $A T M$ autoregulatory feedback mechanism in response to DNA damage, as recently proposed by Clyde et al. [28]. According to these authors, cells have evolved a sensor mechanism that results in rapid induction of the ATM transcript to compensate for ATM chemical inhibition through a negative feedback. In contrast, in our experiments, the decreased expression of ATM transcript could be related to a positive feedback mechanism that down-regulates $A T M$ transcription. 


\subsection{Effects of miR-27a on Proliferation of A549 Cells}

We evaluated the effects of miR-27a over-expression on DDR induced by $\gamma$-rays in A549 cells. In cells enforced to over-express miR-27a, the proliferation rate increased, in particular at $48 \mathrm{~h}$ after transfection, as indicated by the higher number of population doublings ( 2.4 in miR-27a transfected cells vs. $\sim 1.7$ in control cells, Figure 3a). Analysis of cell cycle distribution evidenced perturbations in cell cycle progression of A549 over-expressing miR-27a, as indicated by the increase of cells in S-phase at $48 \mathrm{~h}$ after transfection (31\% vs. 19\%-26\% in control cells, Figure $3 \mathrm{~b}$ ). This finding is in agreement with the results of Lerner et al. [19], showing that miR-27a over-expression significantly increased the number of cells in S-phase in tumor-derived cell lines different from A459. At the same time, we observed an increase of $\mathrm{G}_{2}$ cells from $\sim 7 \%$ in control cells to $\sim 15 \%$ in cells over-expressing miR-27a. We then evaluated if miR-27a up-regulation could influence the colony forming ability of A549 cells. Our experiments show that cloning efficiency was higher in cells over-expressing miR-27a than in control cells (Figure 3c, $p<0.05$ ), indicating that the endogenous level of miR-27a can also affect the cloning efficiency of A549 cells.

Figure 3. Effects of miR-27a up-regulation on proliferation of A549 cells. (a) Number of population doublings in untransfected cells (CTR), cells transfected with miR-27a mimic and mock-transfected cells; (b) Flow cytometric analysis of CTR, mock, and miR-27a transfected cells at $24 \mathrm{~h}$ and $48 \mathrm{~h}$ after transfection; (c) Cloning efficiency determined $24 \mathrm{~h}$ after transfection in cells over-expressing miR-27a, in mock-transfected and untransfected control cells (CTR, * $p<0.05, t$-test).

(a)

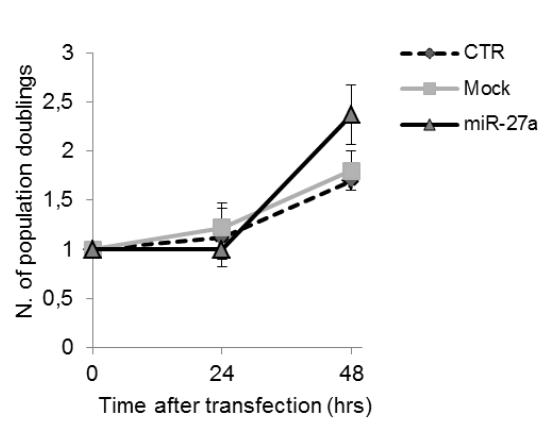

(c)

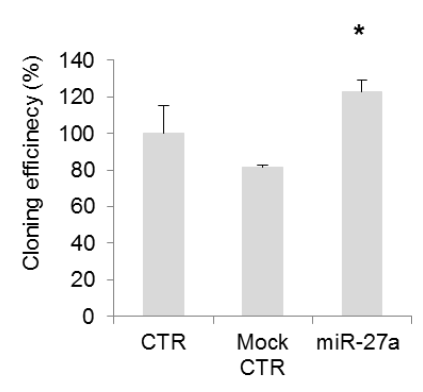

(b)
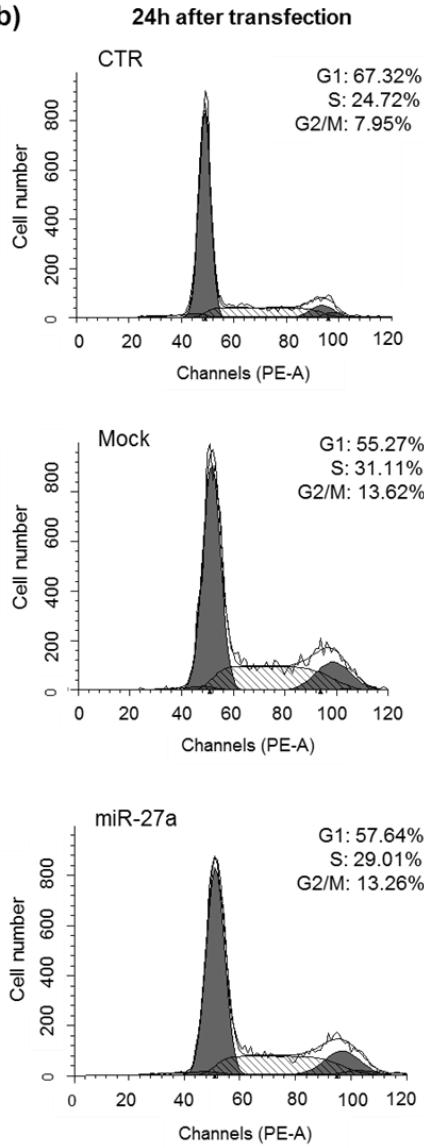
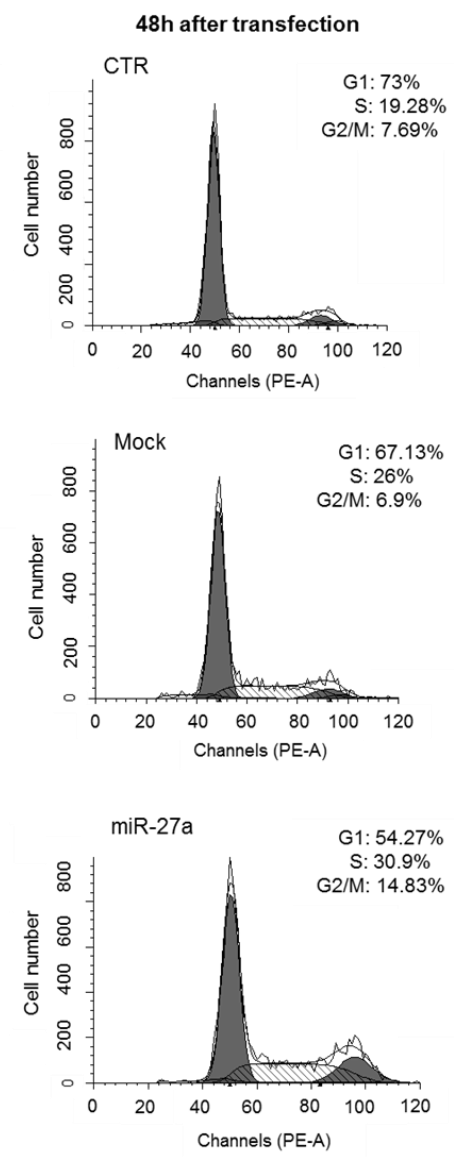
Our findings are, on the whole, consistent with the activity of miR-23a 24-2 27a cluster in promoting cell proliferation as reported for other cancer cells [29,30]. Over-expression of miR-27a has been observed, indeed, to enhance cell proliferation, promote migration and invasion, and activated cell cycling in hepatocellular carcinoma cells [18]. In gastric adenocarcinoma, cell line miR-27a functions as an oncogene and its knocking down could suppress cell growth [17]. Furthermore, miR-27a has been found to promote cell proliferation also in non-cancer cells such as myoblasts through targeting myostatin [31].

\subsection{Effects of miR-27a Up-Regulation on Radiosensitivity of A549 Cells}

In response to DNA DSBs, the serine-threonine protein kinase ATM coordinates many cellular processes, starting with the phosphorylation of numerous substrates active in various branches of the DDR [32,33]. The phosphorylation of $\mathrm{H} 2 \mathrm{AX}(\gamma-\mathrm{H} 2 \mathrm{AX})$ is one of the earliest ATM-dependent responses to IR followed by the formation of nuclear foci with a critical role in the retention of repair factors at the sites of DSBs [34,35]. To examine the effects of miR-27a on ATM signaling in response to IR in A549 cells, we performed the clonogenic survival assay. Our results show that cells over-expressing miR-27a were less sensitive to IR than their counterparts. As shown in Figure 4a, the up-regulation of miR-27a increased cell survival from $25 \%$ to $62 \%$ ( $p<0.05$ ). Our data are in discordance with those reported from breast cancer cells, in which a clonogenic assay showed that ATM down-regulation driven by miR-18a renders cells hypersensitive to IR [36].

Figure 4. Effects of miR-27a up-regulation on radiosensitivity of A549 cells. (a) Cell survival measured by cloning efficiency in control cells (both untransfected cells, CTR, and mock-transfected), in cells subjected to only irradiation (2 Gy), to only transfection with miRNA mimic (miR-27a), and to both transfection with miR-27a mimic and irradiation (miR-27a + 2 Gy); (b) Representative image of clones originated from A549 cells. Error bars represent the mean \pm S.D. $(* p<0.05 ; * * p<0.01 ; * * * p<0.001 ; t$-test).

(a)

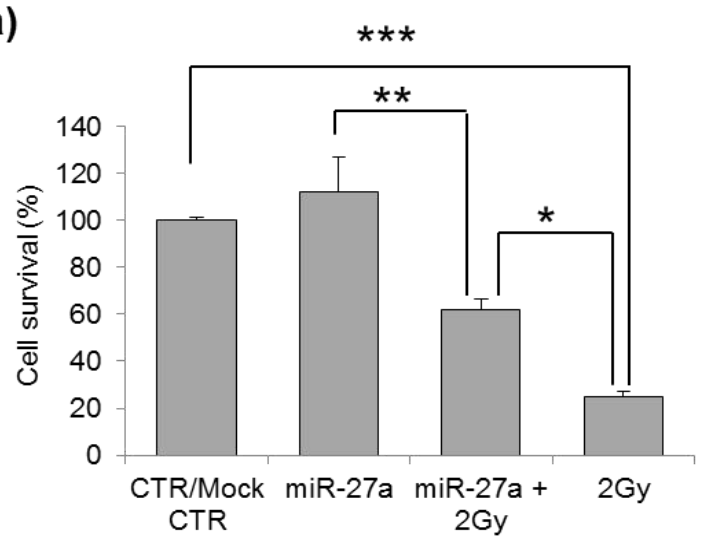

(b)

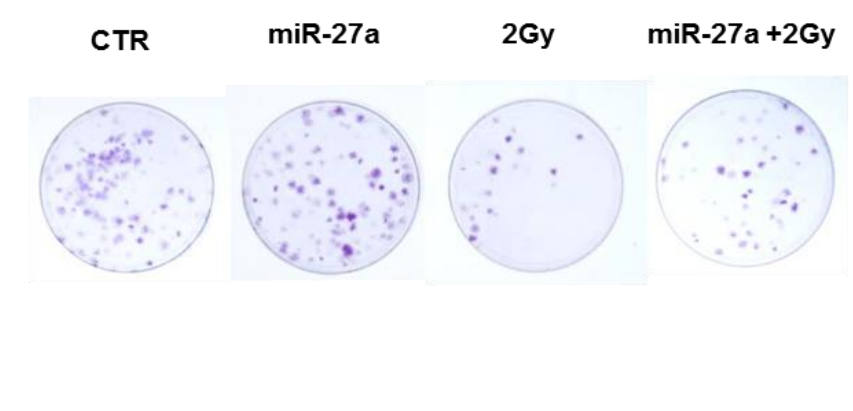

Our results could be consistent with the role of ATM in the control of cell cycle checkpoints, in accordance with data from AT cell lines which showed impaired $G_{1}-S, S$, and $G_{2}-M$ checkpoint arrest following IR exposure [37,38]. Under normal conditions following the induction of DSBs, ATM activates and stabilizes p53, which in turn drives the expression of genes involved in cell proliferation and apoptosis [39,40]. The higher colony forming ability of irradiated A549 cells over-expressing 
miR-27a respect to untransfected irradiated cells could be probably related to alterations in the p53-modulation of genes involved in the activation of cell cycle checkpoints, such as p21. Previous studies have shown, moreover, that heterogeneous nuclear ribonucleoprotein K (hnRNPK) is stabilized in an ATM-dependent manner in response to DNA damage and acts as a cofactor for p53-mediated transcription [41,42]. We thus suggest that when ATM is down-regulated both p53 and hnRNPK activity are affected, inhibiting or altering the p21-mediated cell cycle arrest. To test this hypothesis, we analyzed cell cycle progression by flow cytometry in A549 cells, over-expressing or not miR-27a, after irradiation with 2 Gy of $\gamma$-rays. The results at $6 \mathrm{~h}$ after irradiation show the induction of $\mathrm{G}_{2}$-phase arrest in untransfected cells and in cells over-expressing miR-27a, without differences between the two cells populations (Figure 5a). At $24 \mathrm{~h}$ after irradiation, a little $\mathrm{G}_{2}$ arrest was still present in untransfected cells but not in cells over-expressing the miRNA, without significant differences between the two cells populations (Figure 5b). Flow cytometric analyses carried out on the same samples did not detect a sub-G $\mathrm{G}_{1}$ peak (Figure 5b,d), suggesting that after irradiation with 2 Gy, A549 cells undergo cell cycle arrest rather than apoptosis. According to our findings, the p21-mediated $\mathrm{G}_{2} / \mathrm{M}$ arrest seems unaffected in irradiated A549 cells over-expressing miR-27a, suggesting that the higher colony forming ability of such cells respect to untransfected irradiated cells could be related to a p53-independent mechanism. Indeed, as recently reported by Shin et al. [43], p21 can be activated by both p53-dependent and p53-independent mechanisms and can assume cell cycle arresting functions in response to anti-cancer drugs treatment, depending on the cellular context.

It has been reported that IR could up-regulate ATM expression and consequently lead to phosphorylation of H2AX [44]. Since we observed that in irradiated A549 cells, ATM was $\sim 5$-fold induced in untransfected A549 cells and $~ 1.3$-fold induced in cells transfected with miR-27a mimic (Figure $2 b$ ). We analyzed formation and rejoining of DSBs by determining the number of IR-induced foci of $\gamma-\mathrm{H} 2 \mathrm{AX}$ histone in cells that were or were not over-expressing miR-27a. We analyzed the kinetics of $\gamma-\mathrm{H} 2 \mathrm{AX}$ foci in A549 cells that were or were not over-expressing miR-27a, at $0.5 \mathrm{~h}, 2 \mathrm{~h}$ and $6 \mathrm{~h}$ after irradiation with 2 Gy of $\gamma$-rays, by determining the number of foci positive cells. The kinetics of DSB rejoining appeared very similar in both miR-27a-transfected cells and mock control cells, with a peak of foci induction at $30 \mathrm{~min}$ after irradiation followed by a decrease within $6 \mathrm{~h}$ from irradiation (Figure 6a). At 30 min after irradiation, however, we found that the mean number of $\gamma$-H2AX foci per nucleus was significantly lower in cells over-expressing miR-27a $(p<0.01)$, whereas at later time points, no differences were detected between transfected and untransfected cells (Figure 6b). Our results are in accordance with the reduced $\gamma-\mathrm{H} 2 \mathrm{AX}$ foci formation in response to radiation observed at early post-irradiation times in ATM deficient cells compared with cells expressing wild-type ATM $[44,45]$. 
Figure 5. Flow cytometry analysis of cell cycle distribution of A549 cells at $6 \mathrm{~h}(\mathbf{a}, \mathbf{c})$ and $24 \mathrm{~h}(\mathbf{b}, \mathbf{d})$ after $\gamma$-radiation. (CTR, untransfected non-irradiated cells; $2 \mathrm{~Gy}$, untransfected irradiated cells; miR-27a, cells transfected with miR-27a; miR-27a + 2 Gy, cells transfected with miR-27a and irradiated $24 \mathrm{~h}$ later). (a) Fraction of cells in $\mathrm{G}_{1^{-}}, \mathrm{S}-$ and $\mathrm{G}_{2}$-phase at $6 \mathrm{~h}$ after irradiation and in non-irradiated control cells. The fraction of $\mathrm{G}_{2}$ cells was significantly higher both in miR-27a transfected and untransfected cells, respect to their proper non-irradiated control cells $(26 \% v s .13 \%$, respectively, $* * p<0.01, t$-test); (b) Fraction of cells in $\mathrm{G}_{1^{-}}, \mathrm{S}$ - and $\mathrm{G}_{2}$-phase at $24 \mathrm{~h}$ after irradiation in comparison with their proper non-irradiated control cells. A little $\mathrm{G}_{2}$ arrest was present in untransfected irradiated cells $(10.7 \%$ in 2 Gy cells $v s .7 .4 \%$ in CTR cells, $* p<0.05, t$-test). Representative histogram plots of cell cycle distribution at $6 \mathrm{~h} \mathrm{(c)} \mathrm{and} \mathrm{at} 24 \mathrm{~h}(\mathbf{d})$ after IR are shown. Mock-transfected cells have a cell cycle distribution similar to that of CTR cells at both time points (not shown).

(a)

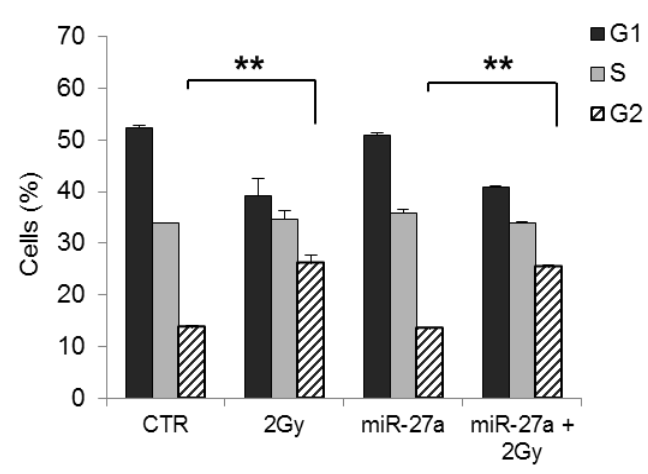

(c)

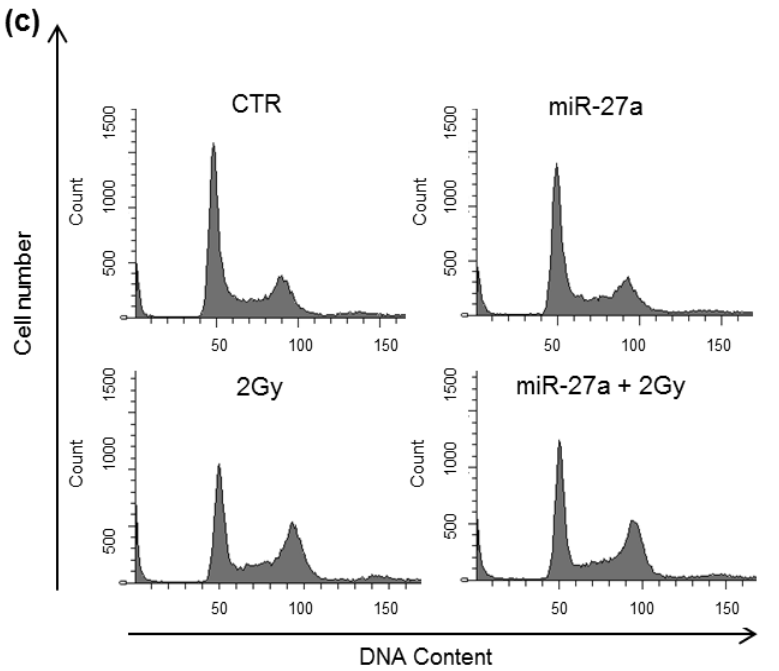

(b)

$24 h$

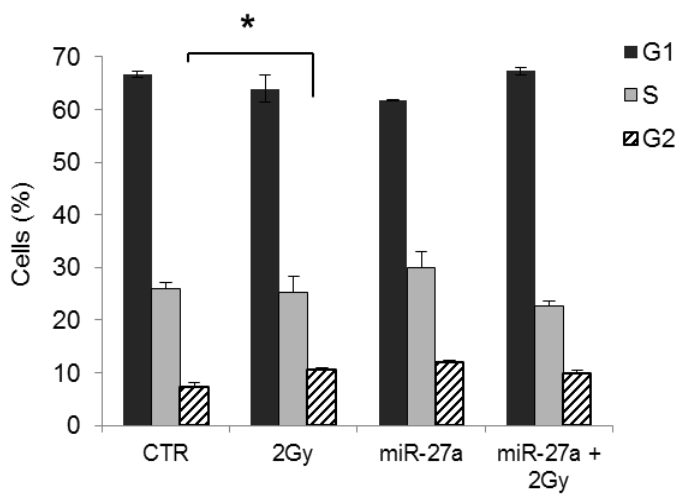

(d)

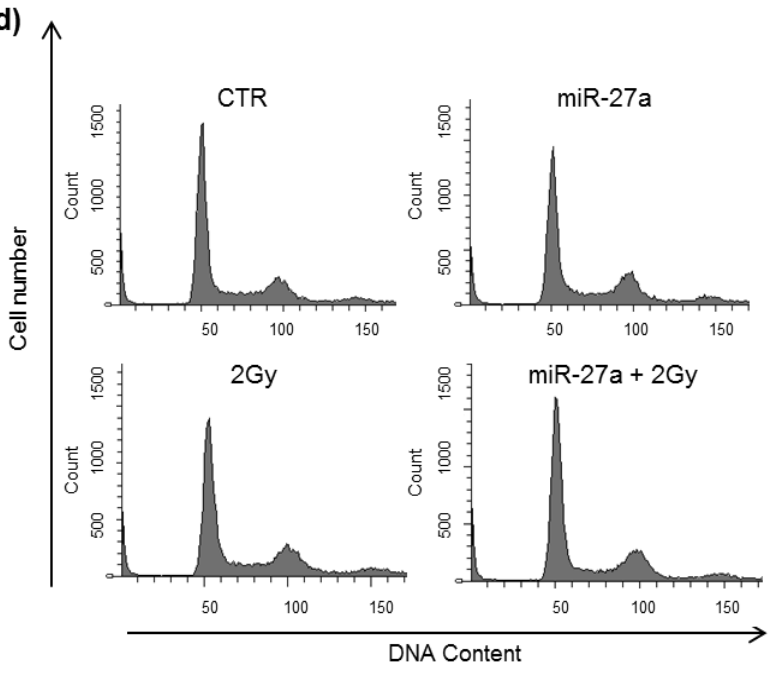


Figure 6. Kinetics of DSB rejoining determined by the percentage of $\gamma-\mathrm{H} 2 \mathrm{AX}$ foci positive cells, over-expressing or not miR-27a, irradiated with 2 Gy. (a) Fraction of A549 cells positive for $\gamma$-H2AX foci at $0.5 \mathrm{~h}, 2 \mathrm{~h}$ and $6 \mathrm{~h}$ after irradiation with $2 \mathrm{~Gy}$; (b) Mean number of $\gamma$-H2AX foci in 2 Gy-irradiated cells over-expressing or not miR-27a, together with representative pictures of foci in nuclei stained with DAPI at $0.5 \mathrm{~h}, 2 \mathrm{~h}$ and $6 \mathrm{~h}$ after $\gamma$-irradiation; quantification of foci/nucleus is from at least 200 cells/time point. Error bars represent the mean \pm S.D. $(* * * p<0.001, t$-test $)$.

(a)

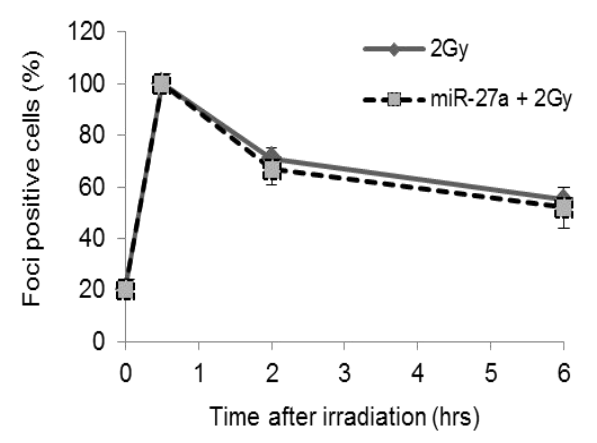

(b)

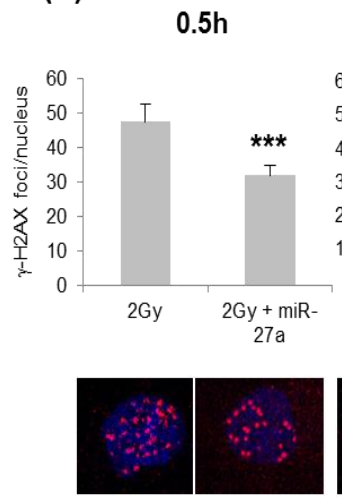

$2 \mathrm{~h}$
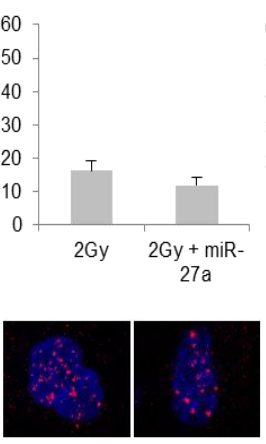

$6 \mathrm{~h}$

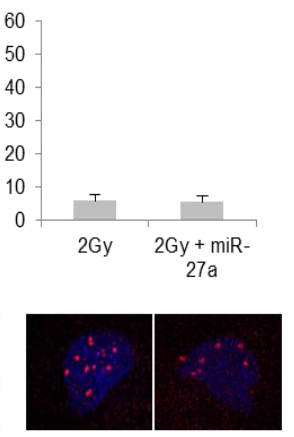

\section{Experimental Section}

\subsection{Cell Culture}

The human A549 cells (lung adenocarcinoma) were purchased from American Type Culture Collection (ATCC n. CCL-185 ${ }^{\mathrm{TM}}$ ) and cultured in Ham's F12-K Nutrient Mixture (Invitrogen Life Technologies, Carlsbad, CA, USA) supplemented with $10 \%$ heat inactivated fetal bovine serum (FBS, BIOCHROM, Berlin, Germany), 38 units $/ \mathrm{mL}$ streptomycin, and 100 units $/ \mathrm{mL}$ penicillin $\mathrm{G}$, in $\mathrm{T} 75 \mathrm{~cm}^{2}$ flasks (FALCON). Cells were kept at $37{ }^{\circ} \mathrm{C}$ in a humidified atmosphere of $95 \%$ air and $5 \% \mathrm{CO}_{2}$, and maintained in exponential and asynchronous phase of growth by repeated trypsinization and reseeding prior to reaching subconfluency.

\subsection{Construction of Recombinant Vectors and Site-Directed Mutagenesis}

Luciferase reporter vectors were generated from human cDNA and cloned into the pmirGLO Dual-Luciferase miRNA Target Expression Vector (Promega, Madison, WI, USA), immediately downstream from the stop codon of the luciferase gene. To predict base-pairing, we used PITA algorithm [46], which predicts the targets of a miRNA by searching for the presence of 6-mer or 7-mer sites that near-perfect match the seed region of the miRNA, allowing for G:U wobbles and considering the role of site accessibility in microRNA target recognition. When indicated, the ATM-3'UTR was mutagenized at the miR-27a recognition sites using the Quick Change Site-Directed Mutagenesis kit (Stratagene, Agilent Technologies, Santa Clara, CA, USA) according to manufacturer's instructions. miR-27a sensor was obtained by annealing, purifying and cloning short oligonucleotides containing three perfect miR-27a binding sites into the SacI and XbaI sites of the pmirGLO vector (Figure 1b). Primers used for the cloning of ATM wild type and mutated were: 


\section{ATM Fwd-5'-ATCTAGGAGCTCAGGAGTGGAAGAAGGCACTG-3'}

ATM Rev-5'-ATCTAGTCTAGAACGCTGTCCAAAGTTTTTCC-3'

ATMdel1 Fwd-5'-AGTGGAAGAAGGCACTCTCAGTGTTGGTGGAC-3'

ATMdel1 Rev-5'-GTCCACCAACACTGAGAGTGCCTTCTTCCACT-3'

ATMdel2 Fwd-5'-CAAGGACAAATGAGGAGTAGTTAGATGAAAATATTAATCATAGAATAGTTGTT-3'

ATMdel2 Rev-5'-AACAACTATTCTATGATTAATATTTTCATCTAACTACTCCTCATTTGTCCTTG-3'

\subsection{Transient Transfection and Cell Irradiation}

Twenty-four hours prior to transfection, cells were plated in $3.5-\mathrm{cm}$ culture dishes at $40 \%-60 \%$ confluence. A549 cells were transfected with pre-miR ${ }^{\text {TM }}$ miRNA Precursor hsa-miR-27a (PM10939, Ambion Austin, TX, USA) by using Lipofectamine ${ }^{\mathrm{TM}} 2000$ (Invitrogen Life Technologies, Carlsbad, CA, USA) for luciferase assays, or Hiperfect Transfection Reagent (QIAGEN, Hilden, Germany) for miRNA over-expression, according to manufacturer's protocol. Mock-transfected cells underwent the transfection process without addition of miRNA (i.e., cells were treated with transfection reagent only). The medium was replaced $4-6 \mathrm{~h}$ after transfection with new culture medium. Transfections were performed in triplicate for each experiment and repeated 3-4 times. Cells were tested for miR or gene over-expression $24 \mathrm{~h}$ later.

Cell irradiation with $\gamma$-rays was performed at $24 \mathrm{~h}$ after transfection at the Department of Oncological and Surgical Sciences of Padova University with a ${ }^{137}$ Cs source. miR-27a-transfected and untransfected cells were irradiated with $2 \mathrm{~Gy}$ (dose rate of $2.8 \mathrm{~Gy} / \mathrm{min}$ ). After irradiation, the medium was replaced with a fresh culture medium.

\subsection{Total RNA Isolation and $q R T-P C R$}

Total RNA was isolated by using Trizol ${ }^{\circledR}$ Reagent (Invitrogen, Life Technologies, Carlsbad, CA, USA), according to the manufacturer's protocol. Total RNA quantification was performed using the ND-1000 spectrophotometer (Nanodrop, Wilmington, DE, USA); RNA integrity and the content of miRNAs were assessed by capillary electrophoresis using the Agilent Bioanalyzer 2100, with the RNA 6000 Nano and the small RNA Nano chips, respectively (Agilent Technologies, Palo Alto, CA, USA).

The endogenous levels of mature miR-27a in untreated and in transfected A549 cells were determined by qRT-PCR using TaqMan MicroRNA Assay kit (Applied Biosystems, Foster City, CA, USA), that incorporate a target-specific stem-loop reverse transcription primer to provide specificity for the mature miRNA target. In brief, each RT reaction $(15 \mathrm{~mL})$ contained $10 \mathrm{ng}$ of total purified RNA, stem-loop RT primer, RT buffer, $0.25 \mathrm{mM}$ each of dNTPs, $50 \mathrm{U}$ MultiScribe ${ }^{\mathrm{TM}}$ reverse transcriptase and 3.8 U RNase inhibitor. The reactions were incubated in a Mastercycler EP gradient S (Eppendorf, Hamburg, Germany) in $0.2 \mathrm{~mL}$ PCR tubes for $30 \mathrm{~min}$ at $16{ }^{\circ} \mathrm{C}, 30 \mathrm{~min}$ at $42{ }^{\circ} \mathrm{C}$, followed by $5 \mathrm{~min}$ at $85{ }^{\circ} \mathrm{C}$, and then held at $4{ }^{\circ} \mathrm{C}$. The resulting cDNA was quantitatively amplified in 40 cycles on an ABI 7500 Real-Time PCR System, using TaqMan Universal PCR Master Mix and TaqMan MicroRNA Assays for miR-27a, and for U48 small nuclear (RNU48) as endogenous control.

For mRNA detection of ATM $1 \mu \mathrm{g}$ of total RNA was retrotranscribed with ImProm-II Reverse Transcription System (Promega, Madison, WI, USA). qRT-PCR was performed with the Go Taq qPCR Master Mix (Promega, Madison, WI, USA) and gene-specific primers for ATM and GAPDH as 
reference. The relative expression levels of ATM and miR-27a were calculated using the comparative delta $\mathrm{Ct}$ (threshold cycle number) method (2-ddCt) implemented in the 7500 Real Time PCR System software (Applied Biosystems ${ }^{\circledR} 7500$ Real-Time PCR System, Life Technologies, Carlsbad, CA, USA, 2007) [47]. qRT-PCR reactions were always performed in quadruplicates.

\subsection{Luciferase Reporter Assays}

A549 cells were plated in 24-well plates $\left(14 \times 10^{5}\right.$ cells/well $)$ and $24 \mathrm{~h}$ later co-transfected with $50 \mathrm{ng}$ of the pmirGLO dual-luciferase constructs, containing the 3'UTR of ATM gene, and with pre-miR ${ }^{\mathrm{TM}}$ miRNA Precursor hsa-miR-27a (miR-27a mimic) or pre-miR ${ }^{\mathrm{TM}}$ miRNA Precursor MoleculesNegative Control (Control mimic) (Ambion, Austin, TX, USA), using Lipofectamine ${ }^{\mathrm{TM}} 2000$ (Invitrogen Life Technologies, Carlsbad, CA, USA). Lysates were collected $24 \mathrm{~h}$ after transfection and Firefly and Renilla Luciferase activities were consecutively measured by using Dual-Luciferase Reporter Assay (Promega, Madison, WI, USA), according to manufacturer's instructions. Relative luciferase activity was calculated by normalizing the ratio of Firefly/Renilla luciferase to that of negative control-transfected cells.

\subsection{Clonogenic Survival Assay}

A549 $\left(2 \times 10^{4}-4 \times 10^{4}\right.$ cells $\left./ \mathrm{cm}^{2}\right)$ were seeded in $3.5-\mathrm{cm}$ culture dishes and allowed to attach overnight, then were subjected to transfection with miR-27a mimic and $24 \mathrm{~h}$ later irradiated with $\gamma$-rays. After irradiation, cells were harvested by trypsinization and counted by trypan blue dye exclusion. 250 viable cells were plated in $6-\mathrm{cm}$ culture dishes in complete medium for the colony-forming assay and grown for 12 days before being stained with crystal violet for colony counting. Cell survival was calculated as percentage of cloning efficiency (CE) of transfected and untransfected cells irradiated with 2 Gy over $\mathrm{CE}$ of their respective non-irradiated control cells (i.e., miR-27a + 2 Gy vs. miR-27a; 2 Gy vs. CTR).

\subsection{Cell Cycle Analysis}

Cells $\left(1 \times 10^{6}\right)$ were harvested, fixed in $70 \%$ cold ethanol and stored at $4{ }^{\circ} \mathrm{C}$ overnight. Before analysis, cells were washed in distilled water, centrifuged and resuspended in $1 \mathrm{~mL}$ PBS containing $50 \mu \mathrm{g} / \mathrm{mL}$ propidium iodide (PI, Sigma-Aldrich, St. Louis, MO, USA) and $100 \mu \mathrm{g} / \mathrm{mL}$ RNAse, for DNA staining. Samples were incubated for $1 \mathrm{~h}$ at $37{ }^{\circ} \mathrm{C}$ and then analyzed using a BD FACSCanto ${ }^{\mathrm{TM}}$ II flow cytometer (BD Biosciences, San Jose, CA, USA). Data from $25 \times 10^{3}$ cells/sample were collected for acquisition and cell cycle distribution analysis using CellQuest (Version 3.0, BD Biosciences, San Jose, CA, USA, 2007) and ModFit LT 3.0 softwares (BD Biosciences, San Jose, CA, USA, 2007), respectively.

\subsection{Immunofluorescence Staining}

A549 cells were grown on glass coverslips for $24 \mathrm{~h}$, transfected with pre-miR-27a and $24 \mathrm{~h}$ later, irradiated. Control cells were subjected to the same treatments except for irradiation. At $0.5 \mathrm{~h}, 2 \mathrm{~h}$, and $6 \mathrm{~h}$ after irradiation, cells were fixed in $4 \%$ formaldehyde (Sigma-Aldrich, St. Louis, MO, USA), 
at $37{ }^{\circ} \mathrm{C}$ for 15 min and washed twice with PBS. The cells were permeabilized with $0.2 \%$ Triton X-100 in PBS at $37{ }^{\circ} \mathrm{C}$ for $10 \mathrm{~min}$ and non-specific binding sites masked with goat serum (10\% in PBS) at room temperature for $1 \mathrm{~h}$. Cells were incubated for $1 \mathrm{~h}$ at room temperature with primary antibody anti- $\gamma$-H2AX (Ser139) (Abcam or Millipore Chemicon, Upstate Clone JBW301, 1:100). After three washes in PBS, cells were incubated with secondary antibody Alexa Fluor 488 goat anti-mouse (Life Technologies, 1:350), washed and counterstained with DAPI $0.2 \mu \mathrm{g} / \mathrm{mL}$.

\subsection{Statistical Analysis}

Data are presented as means \pm standard deviation (S.D.) from two to three independent experiments. All statistical comparisons were carried out by Student's $t$-test and differences with a $p$-value $<0.05$ considered significant.

\section{Conclusions}

In the present work, we examined the role of miR-27a during the DNA-Damage Response (DDR) after irradiation with $\gamma$-rays in A549 cells. The enforced expression of miR-27a in A549 cells altered cell proliferation, by increasing cells in $S$ - and $\mathrm{G}_{2}$-phase. Over-expression of miR-27a decreased the ionizing radiation (IR) toxicity, as evidenced by the higher clonogenic survival and the reduced formation of early $\gamma-\mathrm{H} 2 \mathrm{AX}$ foci in transfected cells compared with the control ones. These findings could be in part related to the action of miR-27a on ATM transcript and in part to the pleiotropic effect of miR-27a on target genes involved in cell proliferation and survival. On the whole, our results indicate that miR-27a promotes proliferation of A549 cells also after irradiation thus decreasing the potency of IR-DDR and probably affecting genome integrity. We cannot exclude, however, that the high basal glucose uptake rates in A549 cells due to the stimulation of epidermal growth factor receptor (EGFR) induced by IR and the EGFR-mediated increase in sodium/glucose cotransport-generated glucose uptake can promote the survival after IR [48] as observed in our experiments.

\section{Acknowledgments}

We gratefully acknowledge Renzo Mazzaro and Leonardo Bee for graphical support. This work was supported by University of Padova (grants 60A06-9009/11; 60A06-3595/12) and by Italian Space Agency (ASI, XMAB-from Molecules to Man, 1/014/06/0).

\section{Conflicts of Interest}

The authors declare no conflict of interest.

\section{References}

1. Harper, J.W.; Elledge, S.J. The DNA damage response: Ten years after. Mol. Cell 2007, 28, 739-745.

2. Stracker, T.H.; Roig, I.; Knobel, P.A.; Marjanović, M. The ATM signaling network in development and disease. Front. Genet. 2013, 4, 37. 
3. Boucas, J.; Riabinska, A.; Jokic, M.; Herter-Sprie, G.S.; Chen, S.; Höpker, K.; Reinhardt, H.C. Posttranscriptional regulation of gene expression-adding another layer of complexity to the DNA damage response. Front. Genet. 2012, 3, 159.

4. Bartel, D.P. microRNAs: Target recognition and regulatory functions. Cell 2009, 136, 215-233.

5. Lee, H.-J. Exceptional stories of microRNAs. Exp. Biol. Med. 2013, 238, 339-343.

6. Bentwich, I.; Avniel, A.; Karov, Y.; Aharonov, R.; Gilad, S.; Barad, O.; Barzilai, A.; Einat, P.; Einav, U.; Meiri, E.; et al. Identification of hundreds of conserved and nonconserved human microRNAs. Nat. Genet. 2005, 37, 766-770.

7. Yu, F.; Yao, H.; Zhu, P.; Zhang, X.; Pan, Q.; Gong, C.; Huang, Y.; Hu, X.; Su, F.; Lieberman, J.; Song, E. Let-7 regulates self renewal and tumorigenicity of breast cancer cells. Cell 2007, 131, 1109-1123.

8. Bae, H.J.; Noh, J.H.; Kim, J.K.; Eun, J.W.; Jung, K.H.; Kim, M.G.; Chang, Y.G.; Shen, Q.; Kim, S.J.; Park, W.S.; et al. microRNA-29c functions as a tumor suppressor by direct targeting oncogenic SIRT1 in hepatocellular carcinoma. Oncogene 2013, doi:10.1038/onc.2013.216.

9. Bisso, A.; Faleschini, M.; Zampa, F.; Capaci, V.; de Santa, J.; Santarpia, L.; Piazza, S.; Cappelletti, V.; Daidone, M.; Agami, R.; et al. Oncogenic miR-181a/b affect the DNA damage response in aggressive breast cancer. Cell Cycle 2013, 12, 1679-1687.

10. Wang, H.F.; Chen, H.; Ma, M.W.; Wang, J.A.; Tang, T.T.; Ni, L.S.; Yu, J.L.; Li, Y.Z.; Bai, B.X. miR-573 regulates melanoma progression by targeting the melanoma cell adhesion molecule. Oncol. Rep. 2013, 30, 520-526.

11. He, L.; He, X.; Lowe, S.W.; Hannon, G.J. microRNAs join the p53 network-Another piece in the tumour-suppression puzzle. Nat. Rev. Cancer 2007, 7, 819-822.

12. Suzuki, H.I.; Yamagata, K.; Sugimoto, K.; Iwamoto, T.; Kato, S. Modulation of microRNA processing by p53. Nature 2009, 460, 529-533.

13. Wouters, M.D.; van Gent, D.C.; Hoeijmakers, J.H.; Pothof, J. microRNAs, the DNA damage response and cancer. Mutat. Res. 2011, 717, 54-66.

14. Girardi, C.; de Pittà, C.; Casara, S.; Sales, G.; Lanfranchi, G.; Celotti, L.; Mognato, M. Analysis of miRNA and mRNA expression profiles highlights alterations in ionizing radiation response of human lymphocytes under modeled microgravity. PLoS One 2012, 7, e31293.

15. Guttilla, I.K.; White, B.A. Coordinate regulation of FOXO1 by miR-27a, miR-96, and miR-182 in breast cancer cells. J. Biol. Chem. 2009, 284, 23204-23216.

16. Gottardo, F.; Liu, C.G.; Ferracin, M.; Calin, G.A.; Fassan, M.; Bassi, P.; Sevignani, C.; Byrne, D.; Negrini, M.; Pagano, F.; et al. micro-RNA profiling in kidney and bladder cancers. Urol. Oncol. 2007, 25, 387-392.

17. Liu, T.; Tang, H.; Lang, Y.; Liu, M.; Li, X. microRNA-27a functions as an oncogene in gastric adenocarcinoma by targeting prohibitin. Cancer Lett. 2009, 273, 233-242.

18. Wu, X.J.; Li, Y.; Liu, D.; Zhao, L.D.; Bai, B.; Xue, M.H. miR-27a as an oncogenic microRNA of hepatitis B virus-related hepatocellular carcinoma. Asian Pac. J. Cancer Prev. 2013, 14, 885-889.

19. Lerner, M.; Lundgren, J.; Akhoondi, S.; Jahn, A.; Ng, H.F.; Akbari Moqadam, F.; Oude Vrielink, J.A.; Agami, R.; den Boer, M.L.; Grandér, D.; et al. miRNA-27a controls FBW7/hCDC4-dependent cyclin E degradation and cell cycle progression. Cell Cycle 2011, 10, 2172-2183. 
20. Volinia, S.; Calin, G.A.; Liu, C.G.; Ambs, S.; Cimmino, A.; Petrocca, F.; Visone, R.; Iorio, M.; Roldo, C.; Ferracin, M.; et al. A microRNA expression signature of human solid tumors defines cancer gene targets. Proc. Natl. Acad. Sci. USA 2006, 103, 2257-2261.

21. Kozaki, K.; Imoto, I.; Mogi, S.; Omura, K.; Inazawa, J. Exploration of tumor-suppressive microRNAs silenced by DNA hypermethylation in oral cancer. Cancer Res. 2008, 68, 2094-2105.

22. Heegaard, N.H.; Schetter, A.J.; Welsh, J.A.; Yoneda, M.; Bowman, E.D.; Harris, C.C. Circulating micro-RNA expression profiles in early stage nonsmall cell lung cancer. Int. J. Cancer 2012, 130, 1378-1386.

23. Chhabra, R.; Dubey, R.; Saini, N. Cooperative and individualistic functions of the microRNAs in the miR-23a 27a 24-2 cluster and its implication in human diseases. Mol. Cancer 2010, 9, 232.

24. Li, X.; Liu, X.; Xu, W.; Zhou, P.; Gao, P.; Jiang, S.; Lobie, P.E.; Zhu, T. C-MYC regulated miR-23a 24-2 27a cluster promotes mammary carcinoma cell invasion and hepatic metastasis by targeting Sprouty2. J. Biol. Chem. 2013, 288, 18121-18133.

25. Zhou, Q.; Gallagher, R.; Ufret-Vincenty, R.; Li, X.; Olson, E.N.; Wang, S. Regulation of angiogenesis and choroidal neovascularization by members of microRNA-23 27 24 clusters. Proc. Natl. Acad. Sci. USA 2011, 108, 8287-8292.

26. Mansour, W.Y.; Bogdanova, N.V.; Kasten-Pisula, U.; Rieckmann, T.; Köcher, S.; Borgmann, K.; Baumann, M.; Krause, M.; Petersen, C.; Hu, H.; et al. Aberrant overlexpression of miR-421 downregulates ATM and leads to a pronounced DSB repair defect and clinical hypersensitivity in SKX squamous cell carcinoma. Radiother. Oncol. 2013, 106, 147-154.

27. Ghosh, S.; Bhat, N.N.; Santra, S.; Thomas, R.G.; Gupta, S.K.; Choudhury, R.K.; Krishna, M. Low energy proton beam induces efficient cell killing in A549 lung adenocarcinoma cells. Cancer Invest. 2010, 28, 615-622.

28. Clyde, R.G.; Craig, A.L.; de Breed, L.; Bown, J.L.; Forrester, L.; Vojtesek, B.; Smith, G.; Hupp, T.; Crawford, J. A novel ataxia-telengiectasia mutated autoregolatory feedback mechanism in murine embryonic stem cells. J. R. Soc. Interface 2009, 6, 1167-1177.

29. Mertens-Talcott, S.U.; Chintharlapalli, S.; Li, X.; Safe, S. The oncogenic microRNA-27a targets genes that regulate specificity protein transcription factors and the G2-M checkpoint in MDA-MB-231 breast cancer cells. Cancer Res. 2007, 67, 11001-11011.

30. Xu, W.; Liu, M.; Peng, X.; Zhou, P.; Zhou, J.; Xu, K.; Xu, H.; Jiang, S. miR-24-3p and miR-27a-3p promote cell proliferation in glioma cells via cooperative regulation of MXI1. Int. J. Oncol. 2013, 42, 757-766.

31. Huang, Z.; Chen, X.; Yu, B.; He, J.; Chen, D. microRNA-27a promotes myoblast proliferation by targeting myostatin. Biochem. Biophys. Res. Commun. 2012, 423, 265-269.

32. Derheimer, F.A.; Kastan, M.B. Multiple roles of ATM in monitoring and maintaining DNA integrity. FEBS Lett. 2010, 584, 3675-3681.

33. Lavin, M.F.; Kozlov, S. ATM activation and DNA damage response. Cell Cycle 2007, 6, 931-942.

34. Celeste, A.; Petersen, S.; Romanienko, P.J.; Fernandez-Capetillo, O.; Chen, H.T.; Sedelnikova, O.A.; Reina-San-Martin, B.; Coppola, V.; Meffre, E.; Difilippantonio, M.J.; Redon, C.; et al. Genomic instability in mice lacking histone H2AX. Science 2002, 296, 922-927. 
35. Celeste, A.; Fernandez-Capetillo, O.; Kruhlak, M.J.; Pilch, D.R.; Staudt, D.W.; Lee, A.; Bonner, R.F.; Bonner, W.M.; Nussenzweig, A. Histone H2AX phosphorylation is dispensable for the initial recognition of DNA breaks. Nat. Cell Biol. 2003, 5, 675-679.

36. Song, L.; Lin, C.; Wu, Z.; Gong, H.; Zeng, Y.; Wu, J.; Li, M.; Li, J. miR-18a impairs DNA damage response through downregulation of ataxia telangiectasia mutated (ATM) kinase. PLoS One 2011, 6, e25454.

37. Beamish, H.; Lavin, M.F. Radiosensitivity in ataxia-telangiectasia: Anomalies in radiation-induced cell cycle delay. Int. J. Radiat. Biol. 1994, 65, 175-184.

38. Rotman, G.; Shiloh, Y. The ATM gene and protein: Possible roles in genome surveillance, checkpoint controls and cellular defense against oxidative stress. Cancer Surv. 1997, 29, 285-304.

39. Rotman, G.; Shiloh, Y. ATM: From gene to function. Hum. Mol. Genet. 1998, 7, 1555-1563.

40. Shiloh, Y.; Ziv, Y. The ATM protein kinase: Regulating the cellular response to genotoxic stress, and more. Nat. Rev. Mol. Cell Biol. 2013, 14, 197-210.

41. Moumen, A.; Masterson, P.; O’Connor, M.J.; Jackson, S.P. hnRNP K: An HDM2 target and transcriptional coactivator of p53 in response to DNA damage. Cell 2005, 123, 1065-1078.

42. Moumen, A.; Magill, C.; Dry, K.L.; Jackson, S.P. ATM-dependent phosphorylation of heterogeneous nuclear ribonucleoprotein $\mathrm{K}$ promotes $\mathrm{p} 53$ transcriptional activation in response to DNA damage. Cell Cycle 2013, 12, 698-704.

43. Shin, D.Y.; Sung Kang, H.; Kim, G.Y.; Kim, W.J.; Yoo, Y.H.; Choi, Y.H. Decitabine, a DNA methyltransferases inhibitor, induces cell cycle arrest at G2/M phase through p53-independent pathway in human cancer cells. Biomed. Pharmacother. 2013, 67, 305-311.

44. Stiff, T.; O’Driscoll, M.; Rief, N.; Iwabuchi, K.; Löbrich, M.; Jeggo, P.A. ATM and DNA-PK function redundantly to phosphorylate $\mathrm{H} 2 \mathrm{AX}$ after exposure to ionizing radiation. Cancer Res. 2004, 64, 2390-2396.

45. Shiloh, Y. The ATM-mediated DNA-damage response: Taking shape. Trends Biochem. Sci. 2006, 31, 402-410.

46. Kertesz, M.; Iovino, N.; Unnerstall, U.; Gaul, U.; Segal, E. The role of site accessibility in microRNA target recognition. Nat. Genet. 2007, 39, 1278-1284.

47. Livak, K.J.; Schmittgen, T.D. Analysis of relative gene expression data using real-time quantitative PCR and the 2(-Delta Delta C(T)) method. Methods 2001, 25, 402-408.

48. Huber, S.M.; Misovic, M.; Mayer, C.; Rodemann, H.P., Dittmann, K. EGFR-mediated stimulation of sodium/glucose cotransport promotes survival of irradiated human A549 lung adenocarcinoma cells. Radiother. Oncol. 2012, 103, 373-379.

(C) 2013 by the authors; licensee MDPI, Basel, Switzerland. This article is an open access article distributed under the terms and conditions of the Creative Commons Attribution license (http://creativecommons.org/licenses/by/3.0/). 